Pacific

Journal of

Mathematics

SYMMETRIC SPACE VALUED MOMENT MAPS

Matthew LeINGANG

Volume $212 \quad$ No. 1

November 2003 


\title{
SYMMETRIC SPACE VALUED MOMENT MAPS
}

\author{
MATtheW LEINGANG
}

For a compact Lie group $G$, three examples of $G$-spaces which can serve as the target of a moment map are discussed. Abstracting the work of Alekseev, Meinrenken, and Malkin, we cast these theories into a unified framework.

Let $G$ be a compact, connected Lie group, and $M$ a manifold on which $G$ acts. There are several natural $G$-spaces which can be considered as the target of a moment map originating from $G$. The first is the dual $\mathfrak{g}^{*}$ to the Lie algebra of $G$; we say that $M$ is a Hamiltonian $G$-space if $M$ has a $G$ invariant symplectic form $\omega$ and there exists an equivariant map $\Phi: M \rightarrow \mathfrak{g}^{*}$ such that

$$
\iota\left(\xi_{M}\right) \omega=d\langle\Phi, \xi\rangle
$$

for all $\xi \in \mathfrak{g} . \Phi$ is called the moment map [5], [4].

In [3], Alekseev, Meinrenken, and Malkin define a Hamiltonian theory in which the moment map has the group itself as target. Given an invariant inner product $B$ on $\mathfrak{g}, M$ is called $q$-Hamiltonian if there is an invariant two-form $\omega$ and an equivariant map $\Phi: M \rightarrow G$ (again called a moment map) such that

$$
d \omega=\frac{1}{12} \Phi^{*} B(\theta,[\theta, \theta]),
$$

and for all $\xi \in \mathfrak{g}$,

$$
\begin{aligned}
\iota\left(\xi_{M}\right) \omega & =\frac{1}{2} \Phi^{*} B(\theta+\bar{\theta}, \xi) ; \\
\operatorname{ker} \omega_{x} & =\left\{\xi_{M}(x) \mid \xi \in \operatorname{ker}\left(\operatorname{Ad}_{\Phi(x)}+1\right)\right\} .
\end{aligned}
$$

Here $\theta$ and $\bar{\theta}$ are the left- and right-invariant Maurer-Cartan forms on $G$. This theory is more complicated, especially when $G$ is nonabelian. For (2a) requires that $\omega$ may not be closed and $(2 \mathrm{~b})$ requires that $\omega$ may not be nondegenerate.

If $G$ is given the structure of a Poisson-Lie group, one can also consider the class of Poisson-Lie $G$-spaces [11], [10]. These have a symplectic form $\omega$ and equivariant map $\Phi: M \rightarrow G^{*}$ such that

$$
\iota\left(\xi_{M}\right) \omega=\Phi^{*}\left\langle\bar{\theta}_{G^{*}}, \xi\right\rangle
$$

for all $\xi$. Here $\bar{\theta}_{G^{*}}$, the right-invariant Maurer-Cartan form on $G^{*}$, takes values in $\mathfrak{g}^{*}$, and hence pairs with $\mathfrak{g}$. The complications here are that the 
individual maps $g: M \rightarrow M$ do not preserve the full structure of $M$, i.e., the Poisson structure, as they do in the two cases above. Rather, the action map itself $G \times M \rightarrow M$ is a Poisson map. However, in [1], Alekseev introduces another target: The canonical noncompact symmetric space $Y$ within $G_{\mathbb{C}}$ transverse to $G$. Let $i: Y \rightarrow G_{\mathbb{C}}$ be the inclusion. The differential equations a moment map $\Phi: M \rightarrow Y$ must satisfy are

$$
d \omega=\frac{1}{2} \Phi^{*} i^{*} \operatorname{Im} B\left(\bar{\theta}_{G_{\mathbb{C}}},\left[\bar{\theta}_{G_{\mathbb{C}}}, \bar{\theta}_{G_{\mathbb{C}}}\right]\right) ;
$$

and for all $\xi$,

$$
\begin{aligned}
\iota\left(\xi_{M}\right) \omega & =\frac{1}{2 \sqrt{-1}} \Phi^{*} i^{*} B\left(\theta_{G_{\mathbb{C}}}+\bar{\theta}_{G_{\mathbb{C}}}, \xi\right) ; \\
\operatorname{ker} \omega_{x} & =0 .
\end{aligned}
$$

They are similar to (2a)-(2c). The spaces $G^{*}$ and $Y$ are equivariantly diffeomorphic, and Alekseev uses $Y$ to construct a correspondence between ordinary Hamiltonian $G$-spaces and Poisson-Lie $G$-spaces. Both $G^{*}$ and $Y$ are equivariantly diffeomorphic to a slightly more natural space, $G_{\mathbb{C}} / G$.

Thus we have three moment map theories, or at least three natural $G$ spaces which serve as targets for moment maps: $\mathfrak{g}^{*}$ for the classical Hamiltonian theory; $G$ for the $q$-Hamiltonian theory, and $G_{\mathbb{C}} / G$ representing the Poisson or $Y$-valued theory. In this note we bring these theories into a unified framework. Abstracting from [3], we show that given a symmetric pair $(H, G)$, with a special pairing on the Lie algebra of $H$, we may construct an equivariantly-closed three-form on $P=H / G$ and a moment map theory. The three most obvious $P$ which arise this way are $G \rtimes \mathfrak{g}^{*} / G=\mathfrak{g}^{*}$, $(G \times G) / G=G$, and $G_{\mathbb{C}} / G \cong Y$, and the moment map theories we will construct coincide with those which have already arisen in the literature. Furthermore, if $(H, G)$ is one of these special symmetric pairs with $H$ connected and $G$ simply connected, $H / G$ must decompose into a product of smaller such symmetric spaces each one of which is isomorphic to $\mathfrak{k}^{*}, K$, or $K_{\mathbb{C}} / K$ for a subgroup $K$ of $G$.

These results were announced in [8]. Shortly thereafter, similar results were related to the author by Yvette Kosmann-Schwarzbach [2]. The author's preprint eventually developed into [9]. He would like to thank his advisors, Victor Guillemin and Shlomo Sternberg, as well as Eckhard Meinrenken and Chris Woodward for many useful discussions. The reviewer also deserves thanks for thoughtful and detailed feedback.

\section{Definitions.}

1.1. Moment space and moment map. There is a basic notion of a differential equation a moment map should obey, regardless of the target, as well as certain conditions of minimal degeneracy. Here we generalize these requirements. 
Definition 1. A (possibly degenerate) moment space for $G$ is a pair $(P, \widetilde{\chi})$, where $P$ is a $G$-manifold and $\tilde{\chi}$ is an equivariantly closed three-form on $P$.

The form $\tilde{\chi}$ may be called the moment form. Since

$$
\Omega_{G}^{3}(P)=\Omega^{3}(P)^{G} \oplus\left(\Omega^{1}(P) \otimes \mathfrak{g}^{*}\right)^{G},
$$

we can write $\tilde{\chi}$ as $\chi+\tau$, where $\chi \in \Omega^{3}(P)^{G}$ is the invariant piece and $\tau: \mathfrak{g} \rightarrow \Omega^{1}(P)$ is the equivariant piece. For any $G$-manifold $Q$, the vector field generated by $\xi \in \mathfrak{g}$ will be denoted $\xi_{Q}$. The condition that $d_{G} \widetilde{\chi}=0$ can be written as three equations:

$$
d \chi=0,
$$

and for all $\xi \in \mathfrak{g}$,

$$
\begin{aligned}
\iota\left(\xi_{P}\right) \chi & =d \tau(\xi) ; \\
\iota\left(\xi_{P}\right) \tau(\xi) & =0 .
\end{aligned}
$$

Example 1. Let $\phi: \mathfrak{g} \rightarrow \mathfrak{g}^{* *} \subset C^{\infty}\left(\mathfrak{g}^{*}\right)$ be the map $\phi(\xi)(\ell)=\langle\xi, \ell\rangle$. Then an equivariantly closed three-form on $\mathfrak{g}^{*}$ is $\tau_{\mathfrak{g}^{*}}=d_{G} \phi$, which has no invariant part. Written as a map $\mathfrak{g} \rightarrow \Omega^{1}\left(\mathfrak{g}^{*}\right)$, it takes the form

$$
\tau_{\mathfrak{g}^{*}}(\xi)_{\ell}(\lambda)=\langle\lambda, \xi\rangle,
$$

for each $\xi \in \mathfrak{g}, \ell \in \mathfrak{g}^{*}$, and $\lambda \in T_{\ell} \mathfrak{g}^{*}=\mathfrak{g}^{*}$.

Example 2. As explained in the introduction, the second example of a moment space is $G$ itself. Let $\mathfrak{g}$ have an invariant, positive-definite inner product $B$. Then the form

$$
\tilde{\chi}_{G}(\xi)=\chi_{G}+\tau_{G}(\xi) \stackrel{\text { def }}{=} \frac{1}{12} B\left(\theta_{G},\left[\theta_{G}, \theta_{G}\right]\right)+\frac{1}{2} B\left(\xi, \theta_{G}+\bar{\theta}_{G}\right)
$$

is equivariantly closed. This is a consequence of the Cartan structure equations

$$
\begin{aligned}
& d \theta=-\frac{1}{2}[\theta, \theta] \\
& d \bar{\theta}=\frac{1}{2}[\bar{\theta}, \bar{\theta}] .
\end{aligned}
$$

Example 3. There are two perspectives on the last example of moment space. The first connects with the Poisson-Lie $G$-spaces of Lu and Weinstein. Let $T$ be a maximal torus for $G, \mathfrak{t}$ its Lie algebra, and $\mathfrak{a}=\sqrt{-1} \mathfrak{t} \subset \mathfrak{g}_{\mathbb{C}}$. $\mathfrak{a}$ is the Lie algebra of a subgroup $A \subset G_{\mathbb{C}}$. Let $\mathbf{n}$ be the sum of a set of positive root spaces. Then there is the Iwasawa decomposition of $\mathfrak{g}_{\mathbb{C}}$ :

$$
\begin{aligned}
\mathfrak{g}_{\mathbb{C}} & =\mathfrak{g} \oplus \mathfrak{a} \oplus \mathbf{n} ; \\
G_{\mathbb{C}} & =G A N .
\end{aligned}
$$

Then the imaginary part of $B_{\mathbb{C}}$ restricts to a nondegenerate pairing between $\mathfrak{g}$ and $\mathfrak{a} \oplus \mathbf{n}$, and thus $\mathfrak{a} \oplus \mathbf{n} \cong \mathfrak{g}^{*}$. Call the group $A N$ by $G^{*}$; then $G_{C}=G^{*} G$. 
The groups $G$ and $G^{*}$ are both Poisson-Lie groups, and $G^{*}$ the dual PoissonLie group to $G$. Left multiplication of $G$ on $G_{\mathbb{C}}$ descends to an action of $G$ on $G^{*}$, called the left dressing action.

We do not seek an equivariantly closed three-form on $G^{*}$, the main difficulty arising from the fact that the dressing action does not preserve the Poisson structure of $G^{*}$. However, one may also consider the space $G_{\mathbb{C}} / G$ as a subspace of $G_{\mathbb{C}}$. Set

$$
Y=\left\{h \in G_{\mathbb{C}} \mid \bar{h}=h^{-1}\right\} .
$$

$Y$ is invariant under the adjoint action of $G$ and equivariantly diffeomorphic to $G^{*}$, and $T_{e} Y=\mathfrak{p}$. Let $\theta_{Y}, \bar{\theta}_{Y} \in \Omega^{1}(Y, \mathfrak{p})$ be the restrictions of the Maurer-Cartan forms from $G_{\mathbb{C}}$ to $Y$. Then the form

(9) $\tilde{\chi}_{Y}(\xi)=\chi_{Y}+\tau_{Y}(\xi) \stackrel{\text { def }}{=} \frac{1}{12} \operatorname{Im} B_{\mathbb{C}}\left(\bar{\theta}_{Y},\left[\bar{\theta}_{Y}, \bar{\theta}_{Y}\right]\right)+\frac{1}{2 \sqrt{-1}} B_{\mathbb{C}}\left(\xi, \bar{\theta}_{Y}+\theta_{Y}\right)$ is real and equivariantly closed.

The equivariantly closed three-form allows us to define a moment map.

Definition 2. Let $M$ be a $G$-manifold and $P$ a moment space for $G$. $M$ is called a $P$-Hamiltonian $G$-space if there exists an invariant two-form $\omega \in$ $\Omega^{2}(M)^{G}$ and an equivariant map $\Phi: M \rightarrow P$ such that

$$
d_{G} \omega=-\Phi^{*} \tilde{\chi}
$$

The $P$-Hamiltonian $G$-space $M$ will further be called nondegenerate if in addition

$$
\operatorname{ker} \omega_{x}=\left\{\xi_{M}(x) \mid \xi \in \operatorname{ker} \tau_{\Phi(x)}: \mathfrak{g} \rightarrow T_{\Phi(x)}^{*} P\right\}
$$

for all $x \in M$.

We may write (10) in terms of its components

$$
\begin{aligned}
d \omega & =-\Phi^{*} \chi ; \\
\iota\left(\xi_{M}\right) \omega & =\Phi^{*} \tau(\xi),
\end{aligned}
$$

for all $\xi \in \mathfrak{g}$. For $p \in P, \tau_{p}$ is defined to be the linear map $\mathfrak{g} \rightarrow T_{p}^{*} P$ which takes $\xi \in \mathfrak{g}$ to the evaluation of the one-form $\tau(\xi)$ at the point $p$. In light of $(12 \mathrm{~b})$, we have that for $p \in P$, the fundamental vector fields of all Lie algebra vectors in the kernel of $\tau_{p}$ must annihilate $\omega$. Thus (11) is a condition of minimal degeneracy.

Example 4. To revisit Example 1, the condition (12b) applied to $\widetilde{\chi}_{\mathfrak{g}^{*}}=\tau_{\mathfrak{g}^{*}}$ is precisely (1). Equations (12a) and (11) state that $\omega$ must be closed and nondegenerate, respectively.

Example 5. The conditions on $G$-valued moment maps are also clearly generalized by Definition 2 (in fact, one could say this example motivates the abstract theory). Alekseev-Meinrenken-Malkin show that the moduli 
space of flat $G$-connections on a Riemann surface with $r>0$ boundary components (divided by the action of the restricted gauge group) has the structure of a Hamiltonian $G^{r+2}$-space with $G^{r+2}$-valued moment map.

Example 6. A $G$-space $M$ with Poisson action is called a Poisson-Lie Gspace if there is a $G$-invariant symplectic form $\omega$ and an equivariant map $\Phi$ such that for all $\xi \in \mathfrak{g}$,

$$
\iota\left(\xi_{M}\right) \omega=2 \Phi^{*} \operatorname{Im}\left(\xi, \bar{\theta}_{G^{*}}\right) .
$$

Choosing instead to work with $Y$-valued moment maps, we may apply Definition 2 to $\tilde{\chi}_{Y}$ and we get (4a)-(4c). Alekseev exhibits equivariant diffeomorphisms $G^{*} \cong Y$, and shows that the corresponding moment map theories are isomorphic.

For any moment space $P$, the most immediate candidates for $P$-Hamiltonian $G$-spaces are the orbits $\mathcal{O}$ of $G$. These have a natural inclusion map $i: \mathcal{O} \rightarrow P$. Indeed, $\tilde{\chi}$ induces a two-form on each orbit $\mathcal{O}$. If $p \in \mathcal{O}, T_{p} \mathcal{O}$ is spanned by $\left\{\xi_{P}(p) \mid \xi \in \mathfrak{g}\right\}$, and we define

$$
\omega_{\mathcal{O}}\left(\xi_{P}(p), \eta_{P}(p)\right)=\tau(\xi)_{p}\left(\eta_{P}(p)\right) .
$$

By $(5 \mathrm{c})$, this form is well-defined and alternating, and we immediately see that it satisfies $(12 \mathrm{~b})$. In fact, $\omega_{\mathcal{O}}$ is characterized by the property that $\iota\left(\xi_{M}\right) \omega_{\mathcal{O}}=i^{*} \tau(\xi)$ for all $\xi \in \mathfrak{g}$. We claim $\omega_{\mathcal{O}}$ is $G$-invariant, and this is a consequence of the equivariance of $\tau$. For, given $\xi, \eta \in \mathfrak{g}$ and $\mathfrak{g} \in G$,

$$
\begin{aligned}
\left.g^{*} \omega_{\mathcal{O}}\right|_{p}\left(\xi_{P}(p), \eta_{P}(p)\right) & =\left.\omega_{\mathcal{O}}\right|_{g} p\left(g_{*} \cdot \xi_{P}(p), g_{*} \cdot \eta_{P}(p)\right) \\
& =\left.\omega_{\mathcal{O}}\right|_{g} p\left(\left(\operatorname{Ad}_{g} \xi\right)_{P}(g p), g_{*} \cdot \eta_{P}(p)\right) \\
& =\tau\left(\operatorname{Ad}_{g} \xi\right)_{g p}\left(g_{*} \cdot \eta_{P}(p)\right) \\
& =\tau(\xi)_{p}\left(\left(g^{-1}\right)_{*} \cdot g_{*} \cdot \eta_{P}(p)\right) \\
& =\left.\omega_{\mathcal{O}}\right|_{p}\left(\xi_{P}(p), \eta_{P}(p)\right) .
\end{aligned}
$$

Therefore, by the relation

$$
0=\mathcal{L}_{\xi_{\mathcal{O}}} \omega_{\mathcal{O}}=d \iota\left(\xi_{\mathcal{O}}\right)+\iota\left(\xi_{\mathcal{O}}\right) d \omega_{\mathcal{O}}
$$

we must have that

$$
\begin{aligned}
\iota\left(\xi_{\mathcal{O}}\right) d \omega_{\mathcal{O}} & =-d \iota\left(\xi_{\mathcal{O}}\right) \omega_{\mathcal{O}} \\
& =-d i^{*} \tau(\xi) \\
& =i^{*} d \tau(\xi) \\
& =-i^{*} \iota\left(\xi_{\mathcal{O}}\right) \chi,
\end{aligned}
$$

which verifies the moment condition (12a). We have proved the following:

Proposition 1. Let $P$ be a moment space for $G$. Consider a $G$-orbit $\mathcal{O} \subseteq P$ with two-form $\omega_{\mathcal{O}}$ given by (14) and moment map given by inclusion. Then $\left(\mathcal{O}, \omega_{\mathcal{O}}, i: \mathcal{O} \rightarrow P\right)$ is a P-Hamiltonian $G$-space. 
We incorporate the minimal degeneracy along orbits into our definition.

Definition 3. A moment space $P$ is called nondegenerate if all orbits $\mathcal{O} \subseteq$ $P$ are nondegenerate $P$-Hamiltonian $G$-spaces with two-form given by (14) and moment map inclusion. This means that for all $p \in P$,

$$
\operatorname{ker} \tau_{p} \cap \mathfrak{g}_{p}=\{0\},
$$

and for all $\xi \in \mathfrak{g}$,

$$
\tau(\xi)_{p}\left(\eta_{p}(p)\right)=0 \forall \eta \in \mathfrak{g} \Longrightarrow \tau(\xi)_{p} \equiv 0 .
$$

Lemma 1. Let $M$ be a nondegenerate P-Hamiltonian $G$-space with twoform $\omega$ and moment map $\Phi: M \rightarrow P$. Let $x \in M$. Then:

(a) The map $\xi \mapsto \xi_{M}(x)$, restricted to $\operatorname{ker} \tau_{x} \rightarrow \operatorname{ker} \omega_{x}$, is an isomorphism.

(b) We have ker $\left.\left.d \Phi\right|_{x} \cap \operatorname{ker} \omega\right|_{x}=\{0\}$.

Proof. The first claim is obvious given (15a). For the second, let $v \in$ $\left.\left.\operatorname{ker} d \Phi\right|_{x} \cap \operatorname{ker} \omega\right|_{x}$. Then $v=\xi_{M}(x)$ for some $\xi \in \operatorname{ker} \tau_{\Phi(x)}$. However, since

$$
0=d \Phi(v)=d \Phi\left(\xi_{M}(x)\right)=\xi_{P}(\Phi(x)),
$$

(the last equality is by the equivariance of $\Phi$ ), we must have that $\xi \in \mathfrak{g}_{\Phi(p)}$. Hence again by (15b), we have $\xi=0$.

Proposition 2. Let $M_{1}$ and $M_{2}$ be nondegenerate P-Hamiltonian $G$-spaces and $F: M_{1} \rightarrow M_{2}$ an equivariant map such that $F^{*} \omega_{2}=\omega_{1}$ and $F^{*} \Phi_{2}=\Phi_{1}$. Then $F$ is an immersion.

Proof. Since $F^{*} \omega_{2}=\omega_{1}$, we have that ker $\left.d F\right|_{x}=\left.\operatorname{ker} \omega_{1}\right|_{x}$. Also, since $F^{*} \Phi_{2}=\Phi_{1}$, we have

$$
\left.\left.\operatorname{ker} \omega_{1}\right|_{x} \cong \operatorname{ker} \omega_{2}\right|_{F(x)} \cong \operatorname{ker} \tau_{\Phi_{1}(x)} .
$$

Thus ker $\left.\left.d F\right|_{x} \cap \operatorname{ker} \omega_{1}\right|_{x}=\{0\}$, and therefore $\left.d F\right|_{x}$ is injective.

From this we can prove the "P-Hamiltonian Kostant Theorem."

Theorem 1. Let $M$ a transitive nondegenerate P-Hamiltonian $G$-space. Then the moment map $\Phi: M \rightarrow P$ is a covering map onto an orbit.

Proof. For $x_{0} \in M$, let $\mathcal{O}$ be the orbit of $\Phi\left(x_{0}\right) \in P$. Then since $M$ is transitive, the image of $\Phi$ consists of $\mathcal{O}$ alone. That $\Phi$ is a submersion onto its image is clear since $T_{p} \mathcal{O}=\mathfrak{g}_{P}(p)$ and $\xi_{P}(p)=d \Phi\left(\xi_{M}(x)\right)$ if $p=\Phi(x)$. Finally, we have that $\Phi^{*} \omega_{\mathcal{O}}=\omega$ by (12b) and (14), and applying the previous proposition, $\Phi$ is an immersion as well.

Definition 4. Let $M$ be a $P$-Hamiltonian $G$-space. Suppose that $o \in M$ is a $G$-fixed point. We define the reduced space of $M$ at $o$ to be $M_{o}=$ $\Phi^{-1}(o) / G$. 
$M_{o}$ has a special two-form on it arising from that on $M$. To see this, put $Z=\Phi^{-1}(o)$, and let $i: Z \rightarrow M, \pi: Z \rightarrow M_{o}$ be the inclusion and projection. Denote the $P$-Hamiltonian two-form on $M$ is by $\omega$. It is $G$-invariant, and therefore so it is restriction to $Z$. Furthermore, for $\xi \in \mathfrak{g}$,

$$
\begin{aligned}
\iota\left(\xi_{Z}\right) i^{*} \omega & =i^{*} \iota\left(\xi_{M}\right) \omega \\
& =i^{*} \Phi^{*} \tau(\xi) \\
& =0,
\end{aligned}
$$

since $\Phi \circ i$ is the constant map $o$. Thus $i^{*} \omega$ is $G$-basic; there exists $\omega_{o} \in$ $\Omega^{2}\left(M_{o}\right)$ such that $\pi^{*} \omega_{o}=i^{*} \omega$. Notice also that since

$$
\pi^{*} d \omega_{o}=d \pi^{*} \omega_{o}=d i^{*} \omega=i^{*} d \omega=i^{*} \Phi^{*} \chi=0,
$$

we must have that $d \omega_{o}=0$.

Theorem 2. Let $M$ be a nondegenerate P-Hamiltonian $G$-space. Suppose that $\operatorname{dim} P=\operatorname{dim} G$. Then $\omega_{o}$ is a symplectic form if and only o is a regular value of the moment map $\Phi$.

Proof. Let $z \in Z$. The map $\tau_{o}$ is injective by (15a) and therefore an isomorphism since $\operatorname{dim} P=\operatorname{dim} G$. Thus $\left.\omega\right|_{z}$ is nondegenerate. Therefore, we have a commutative diagram

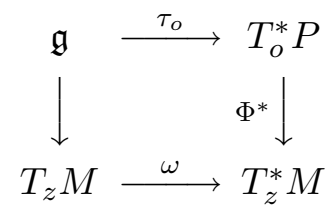

where the horizontal maps are both isomorphisms. It follows from the basic $\left(\operatorname{ker} T^{*}\right)^{0}=\operatorname{im} T$ theorem of linear algebra that

$$
\left(\left.\operatorname{ker} d \Phi\right|_{z}\right)^{\omega}=\mathfrak{g}_{M}(z) .
$$

Now $T_{z} Z \subseteq$ ker $\left.d \Phi\right|_{z}$, by the definition of $Z$ as the inverse image of $o$. Thus we have

$$
T_{z}(G \cdot z)=\mathfrak{g}_{M}(z)=\left(\left.\operatorname{ker} d \Phi\right|_{z}\right)^{\omega} \subseteq\left(T_{z} Z\right)^{i^{*} \omega} .
$$

The left-hand side of the above is the kernel of $\pi_{*}: T_{z} Z \rightarrow T_{\pi(z)} M_{o}$, and the right-hand side is the kernel of $i^{*} \omega_{o}$ at $z$. The two are equal (and $\omega_{o}$ is therefore nondegenerate) if and only equality holds in the last step. This is true if and only if $T_{z} Z=\left.\operatorname{ker} d \Phi\right|_{z}$, i.e., if $z$ is regular.

\section{Manin structure.}

A symmetric pair over $G$ consists of a Lie group $H \supset G$ and an involution $\sigma$ of $H$ such that $H^{\sigma}=G$. Let $\mathfrak{h}$ be the Lie algebra of $H$ and $s$ the derivative of $\sigma$ at the identity. Then $(\mathfrak{h}, s)$ is a symmetric Lie algebra, and $\mathfrak{h}^{\sigma}=\mathfrak{g} \cdot \mathfrak{h}$ 
has a canonical decomposition $\mathfrak{h}=\mathfrak{g} \oplus \mathfrak{p}$, where $\mathfrak{p}$ is the -1 eigenspace of $s$. We have the commutation relations

$$
[\mathfrak{g}, \mathfrak{g}] \subset \mathfrak{g} ; \quad[\mathfrak{g}, \mathfrak{p}] \subset \mathfrak{p} ; \quad[\mathfrak{p}, \mathfrak{p}] \subset \mathfrak{g} .
$$

Let $P=H / G$ where, and $o=e G$. Then $P$ is a symmetric space and $\mathfrak{p}$ is canonically identified with $T_{o} P$. See $[6]$.

We will often take the involution to be understood and refer to the symmetric pair as $(H, G)$. There are three important example of symmetric pairs over $G$.

Example 7. On $H_{0}=G \ltimes \mathfrak{g}^{*}$, the involution $\sigma_{0}$ is the map $(g, \ell) \mapsto(g,-\ell)$. The corresponding symmetric Lie algebra is $\mathfrak{h}_{0}=\mathfrak{g} \ltimes \mathfrak{g}^{*}$ with Lie bracket and involution

$$
\begin{aligned}
{[(\xi, \lambda),(\eta, \mu)] } & =\left([\xi, \eta], \operatorname{ad}_{\xi}^{*} \mu-\operatorname{ad}_{\eta}^{*} \lambda\right) ; \\
s_{0}(\xi, \lambda) & =(\xi,-\lambda) .
\end{aligned}
$$

Example 8. On $H_{+}=G \times G, G$ is embedded as the diagonal. This subgroup is fixed by the involution $\sigma_{+}\left(g_{1}, g_{2}\right)=\left(g_{2}, g_{1}\right)$. The corresponding symmetric Lie algebra is $\mathfrak{h}_{+}=\mathfrak{g} \times \mathfrak{g}$ with involution

$$
\sigma_{+}\left(\xi_{1}, \xi_{2}\right)=\left(\xi_{2}, \xi_{1}\right)
$$

fixing the diagonal subalgebra.

Example 9. Let $G$ be simply connected as well, so that there is a complex, simply connected group $G_{\mathbb{C}}$ with $G$ as its real form. There are the conjugation automorphisms of $G_{\mathbb{C}}$ and $\mathfrak{h}_{-}=\mathfrak{g}_{\mathbb{C}}=\mathfrak{g} \otimes \mathfrak{C}$ singling out the real forms as their fixed point sets.

$$
s_{-}(\xi+\sqrt{-1} \eta)=\xi-\sqrt{-1} \eta .
$$

In order to consider the quotient spaces $H / G$ as moment spaces for $G$, we need to pair elements of $\mathfrak{g}$ with elements of $\mathfrak{p}$. The following structure makes this possible:

Definition 5. Let $(\mathfrak{h}, s)$ be a symmetric Lie algebra. $\mathfrak{h}$ will be called a Manin symmetric Lie algebra if it admits a nondegenerate symmetric bilinear form $q$ with respect to which $s$ is skew-symmetric: I.e., for all $\zeta_{1}, \zeta_{2} \in \mathfrak{h}$ :

$$
q\left(s \zeta_{1}, \zeta_{2}\right)=-q\left(\zeta_{1}, s \zeta_{2}\right) .
$$

The pairing $q$ will be called a Manin form or Manin pairing. It is also assumed to be invariant with respect to the adjoint action of $\mathfrak{h}$ on itself: For all $\zeta_{1}, \zeta_{2}, \zeta_{3} \in \mathfrak{h}$,

$$
\begin{aligned}
q\left(\operatorname{ad}_{\zeta_{1}} \zeta_{2}, \zeta_{3}\right) & =q\left(\zeta_{2}, \operatorname{ad}_{\zeta_{1}} \zeta_{3}\right), \text { or } \\
q\left(\left[\zeta_{1}, \zeta_{2}\right], \zeta_{3}\right) & =q\left(\zeta_{1},\left[\zeta_{2}, \zeta_{3}\right]\right) .
\end{aligned}
$$

Let $(H, G)$ be a symmetric pair. $H$ will be called a Manin symmetric pair if the associated symmetric Lie algebra $\mathfrak{h}$ admits a Manin form which is 
invariant with respect to the adjoint action of $H$ on $\mathfrak{h}$. That is, in addition to (17) and (18), we must have, for all $\zeta_{1}, \zeta_{2} \in \mathfrak{h}$ and $h \in H$,

$$
q\left(\operatorname{Ad}_{h} \zeta_{1}, \zeta_{2}\right)=q\left(\zeta_{1}, \operatorname{Ad}_{h^{-1}} \zeta_{2}\right) .
$$

Proposition 3. Let $G$ be a compact, connected Lie group and $\mathfrak{g}$ its Lie algebra.

(a) $\mathfrak{h}_{0}=\mathfrak{g} \rtimes \mathfrak{g}^{*}$ has a Manin pairing given by

$$
q_{0}\left(\left(\xi_{1}, \lambda_{1}\right),\left(\xi_{2}, \lambda_{2}\right)\right)=\left\langle\xi_{1}, \lambda_{2}\right\rangle+\left\langle\xi_{2}, \lambda_{1}\right\rangle .
$$

$\left(H_{0}=G \rtimes \mathfrak{g}^{*}, G\right)$ is a Manin symmetric pair. The resulting symmetric space is isomorphic to $\mathfrak{g}^{*}$.

(b) Let $\mathfrak{g}$ have an invariant inner product $B . \mathfrak{h}_{+}=\mathfrak{g} \times \mathfrak{g}$ has a Manin pairing given by

$$
q_{+}\left(\left(\xi_{1}, \eta_{1}\right),\left(\xi_{2}, \eta_{2}\right)\right)=\frac{1}{2}\left(B\left(\xi_{1}, \xi_{2}\right)-B\left(\eta_{1}, \eta_{2}\right)\right) .
$$

Since $G$ is connected, $\left(H_{+}=G \times G, \Delta(G)\right)$ is a Manin symmetric pair. The resulting symmetric space is isomorphic to $G$.

(c) Again assume $\mathfrak{g}$ has an inner product $B$. Then $B$ extends to a $\mathbb{C}$ bilinear inner product on $\mathfrak{h}_{-}=\mathfrak{g} \otimes \mathbb{C}$. $\mathfrak{h}_{-}$has a a Manin pairing given by

$$
q_{-}\left(\zeta_{1}, \zeta_{2}\right)=\operatorname{Im} B\left(\zeta_{1}, \zeta_{2}\right)
$$

Proof. Clear.

\section{Construction of the moment form.}

The purpose of this section is to show that given a Manin symmetric pair, we can construct a moment space. This space will in fact be the space of right cosets.

For this section $(H, G)$ will be a Manin symmetric pair with involution $\sigma$ and a Manin pairing $q$. The corresponding involution of $\mathfrak{h}$ will be denoted $s$.

3.1. The equivariant form. Let $\theta$ be the left-invariant Maurer-Cartan form on $H$ taking values in $\mathfrak{h}$. Using $s$, we can decompose $\theta$ into its "g-part" and its "p-part," defining:

$$
\gamma=\frac{1+s}{2} \theta ; \quad \pi=\frac{1-s}{2} \theta,
$$

so $\gamma \in \Omega^{1}(H, \mathfrak{g})$ and $\pi \in \Omega^{1}(H, \mathfrak{p})$. Let $j: H \rightarrow P=H / G$ be the quotient map.

Proposition 4. Define for $\xi \in \mathfrak{g}$ a one-form

$$
\beta(\xi)_{h}=q\left(\xi, \operatorname{Ad}_{h} \pi\right) \in \Omega^{1}(H) .
$$

Then: 
(a) $\beta(\xi)$ is basic with respect to the right action of $G$ on $H$, so there is a unique one-form $\tau(\xi) \in \Omega^{1}(P)$ such that $j^{*} \tau(\xi)=\beta(\xi)$.

(b) The map $\xi \mapsto \beta(\xi)$ is equivariant with respect to the left action of $G$ on $H$, so $\tau$ is an equivariant three-form on $P$.

(c) We have, for all $\xi \in \mathfrak{g}$,

$$
\iota\left(\xi_{P}\right) \tau(\xi)=0,
$$

where $\xi_{P}$ is the vector field on $P$ generated by the left action of $G$ in the direction $\xi$.

Proof. For $h \in H$, let $R_{h}$ and $L_{h}$ denote left and right multiplication by $h$ as diffeomorphisms of $H$. Since $R_{g}^{*} \theta=\operatorname{Ad}_{g^{-1}} \theta$ and $\sigma(g)=g$, it follows that $R_{g}^{*} \pi=\operatorname{Ad}_{g^{-1}} \pi$. Then

$$
\begin{aligned}
\left(R_{g}^{*} \beta(\xi)\right)_{h} & =q\left(\xi, R_{g}^{*} \operatorname{Ad}_{h} \pi\right) \\
& =q\left(\xi, \operatorname{Ad}_{h g} \operatorname{Ad}_{g^{-1}} \pi\right) \\
& =q\left(\xi, \operatorname{Ad}_{h} \pi\right)=\beta(\xi)_{h},
\end{aligned}
$$

so $\beta(\xi)$ is right-invariant. Moreover, if $\eta_{R}(h)=\left(L_{h}\right)_{*} \eta$ is the fundamental vector field associated to the right action corresponding to $\eta$, then $\theta\left(\eta_{R}\right)=\eta$. Hence $\pi\left(\theta_{R}\right)=0$ and

$$
\beta(\xi)_{h}\left(\eta_{R}\right)=0 .
$$

Thus $\beta(\xi)$ is also right-horizontal, hence right-basic. This proves the first claim of the proposition.

For the second, note that $\theta$ and hence $\pi$ are left $H$-invariant, so

$$
\begin{aligned}
\left(L_{g^{-1}}^{*} \beta(\xi)\right)_{h} & =q\left(\xi, \operatorname{Ad}_{g^{-1}} \pi\right) \\
& =q\left(\operatorname{Ad}_{g} \xi, \operatorname{Ad}_{h} \pi\right)=\beta\left(\operatorname{Ad}_{g} \xi\right)_{h} .
\end{aligned}
$$

Finally, to prove the third claim, we will show that for $\xi \in \mathfrak{g}$,

$$
\iota\left(\xi_{L}\right) \beta(\xi)=0,
$$

where $\xi_{L}$ is the fundamental vector field on $H$ associated to the left action. Indeed,

$$
\begin{aligned}
\beta\left(\xi_{h}\right)\left(\xi_{L}\right) & =q\left(\xi, \operatorname{Ad}_{h} \frac{\operatorname{Ad}_{h^{-1}}-\operatorname{Ad}_{\sigma\left(h^{-1}\right)}}{2} \xi\right) \\
& =\frac{1}{2} q(\xi, \xi)-\frac{1}{2}\left(\operatorname{Ad}_{h-1} \xi, \operatorname{Ad}_{\sigma\left(h^{-1}\right)} \xi\right) \\
& =0 .
\end{aligned}
$$

Remark. It is only in (25) that we used the full $\mathrm{Ad}_{H}$-invariance of the pairing $q$. In fact, the first two claims of Proposition 4 can be proven with only a pairing between $\mathfrak{g}$ and $\mathfrak{p}$ which is $\mathrm{Ad}_{G}$-invariant $\left(\right.$ note $\operatorname{Ad}_{G}$ preserves 
the decomposition $\mathfrak{h}=\mathfrak{g} \oplus \mathfrak{p})$. There is a unique extension of such a pairing to an $s$-skew pairing of the full Lie algebra, and to force the associated one-form to obey (24) is to require that

$$
q\left(\operatorname{Ad}_{h} \xi, \eta\right)=q\left(\xi, \operatorname{Ad}_{h^{-1}} \eta\right)
$$

for all $\xi, \eta \in \mathfrak{g}$, and for all $h$ of the form $\sigma(k) k^{-1}$. Such $h$ lie in a submanifold $V=\left\{h \in H \mid \sigma(h)=h^{-1}\right\}$, which is transverse to $G$ at the identity of $H$. In fact $T_{e} V=\mathfrak{p}$.

3.2. The invariant form. Here we will extend $\tau \in \Omega_{G}^{3}(H / G)$ to an equivariantly closed three-form.

Proposition 5. Define $\Xi \in \Omega^{3}(H)$ by

$$
\Xi=\frac{1}{3} q(\pi,[\pi, \pi]) .
$$

Then:

(a) $\Xi$ is right $G$-basic and left $G$-invariant. Hence there exists a unique $\chi \in \Omega^{3}(P)^{G}$ such that $\Xi=j^{*} \chi$.

$$
d \chi=0
$$

(c) For $\xi \in \mathfrak{g}$,

$$
\iota\left(\xi_{P}\right) \chi=d \tau(\xi) .
$$

Proof. Writing $\theta=\gamma+\pi$ as the decomposition of $\theta$ relative to that of $\mathfrak{h}$, we have

$$
\begin{aligned}
& d \gamma=-\frac{1}{2}([\gamma, \gamma]+[\pi, \pi]) ; \\
& d \pi=-[\gamma, \pi] .
\end{aligned}
$$

This is an immediate consequence of the bracket identities for a symmetric Lie algebra (16) and the Cartan structure equation (8a). The proposition reduces to a formal calculation.

(a) This is proved similarly to the analogous claim in Proposition 4.

(b) By the Jacobi identity

$$
[\pi,[\pi, \pi]]=[\theta,[\theta, \theta]]=[\gamma,[\gamma, \gamma]]=0 .
$$

Thus,

$$
\begin{aligned}
d \Xi & =\frac{1}{3} d q(\pi,[\pi, \pi]) \\
& =q(d \pi,[\pi, \pi]) \\
& =-q([\gamma, \pi],[\pi, \pi]) \\
& =-q(\gamma,[\pi,[\pi, \pi]])=0 .
\end{aligned}
$$

So (27) is proved. 
(c) Let $\xi \in \mathfrak{g}$. Then

$$
\begin{aligned}
\iota\left(\xi_{L}\right) \Xi & =\frac{1}{3} \iota\left(\xi_{L}\right) q(\pi,[\pi, \pi]) \\
& =q\left(\pi\left(\xi_{L}\right),[\pi, \pi]\right) \\
& =q\left(\operatorname{Ad}_{h^{-1}} \xi,[\pi, \pi]\right) .
\end{aligned}
$$

On the other hand,

$$
\begin{aligned}
d \beta(\xi) & =d q\left(\xi, \operatorname{Ad}_{h} \pi\right) \\
& =q\left(\xi, \operatorname{Ad}_{h} \operatorname{ad}_{\theta} \pi\right)-q\left(\xi, \operatorname{Ad}_{h}[\pi, \gamma]\right) \\
& =q\left(\xi, \operatorname{Ad}_{h}[\gamma+\pi, \pi]\right)-q\left(\xi, \operatorname{Ad}_{h}[\pi, \gamma]\right) \\
& =q\left(\xi, \operatorname{Ad}_{h}[\pi, \pi]\right) .
\end{aligned}
$$

Thus (28) is true as well.

As an immediate consequence, we have:

Theorem 3. If $(H, G, \sigma, q)$ is a Manin symmetric pair, the equivariant three-form $\tilde{\chi}=\chi+\tau$ is equivariantly closed, thus giving $H / G$ the structure of a moment space for $G$.

3.3. Nondegeneracy. Along with the equivariant condition (Definition 1), which we have just satisfied for an arbitrary Manin symmetric pair, there is the nondegeneracy (actually, minimal degeneracy) condition of Definition 3. Here we will use the nondegeneracy of the pairing to satisfy nondegeneracy of $\tau$.

Proposition 6. Let $(H, G)$ be a Manin symmetric pair, and $\mathcal{O}$ an orbit of $G$ in $P=H / G$. Then $P$ with two-form given by (14) and moment map $i: \mathcal{O} \rightarrow P$ satisfies

$$
\operatorname{ker} \omega_{p}=\left\{\xi_{P}(p) \mid \xi \in \operatorname{ker} \tau_{p}: \mathfrak{g} \rightarrow T_{p}^{*} P\right\} .
$$

Hence $\mathcal{O}$ is a nondegenerate $P$-Hamiltonian $G$-space.

Thus:

Theorem 4. Let $(H, G, \sigma, q)$ be a Manin symmetric pair. Then $P=H / G$ is a nondegenerate moment space for $G$.

Proof. What we are attempting to prove is

$$
\iota\left(\xi_{P}\right) \omega_{p}=0 \Longleftrightarrow\left\{\begin{array}{l}
\xi_{P}(p)=0 \text { or } \\
\xi \in \operatorname{ker} \tau_{p} .
\end{array}\right.
$$

Suppose that $\xi \in \operatorname{ker} \tau_{p}$, where $p=h G$. This means that

$$
0=q\left(\operatorname{Ad}_{h^{-1}} \xi, \frac{1-s}{2} \operatorname{Ad}_{h^{-1}} \eta\right)=-\frac{1}{2} q\left(\operatorname{Ad}_{h^{-1}} \xi, \operatorname{Ad}_{\sigma\left(h^{-1}\right)} \eta\right)
$$


for all $\eta \in \mathfrak{g}$. Since the $q$-orthogonal space to $\mathfrak{g}$ within $\mathfrak{h}$ is $\mathfrak{g}$ itself, we have that

$$
\operatorname{Ad}_{\sigma(h) h^{-1} \xi} \in \mathfrak{g} .
$$

Write $k=\sigma(h) h^{-1}$ and note that $\sigma(k)=k^{-1}$. Then by (32) we must have that

$$
\operatorname{Ad}_{k^{-1}} \xi=\sigma\left(\operatorname{Ad}_{k} \xi\right)=\operatorname{Ad}_{k} \xi
$$

and therefore $\xi=\operatorname{Ad}_{k}^{2} \xi$ or

$$
\xi \in \operatorname{ker}\left(1-\operatorname{Ad}_{k}^{2}\right) .
$$

Now we have a direct sum decomposition

$$
\operatorname{ker}\left(1-\operatorname{Ad}_{k}^{2}\right)=\operatorname{ker}\left(1-\operatorname{Ad}_{k}\right) \oplus \operatorname{ker}\left(1+\operatorname{Ad}_{k}\right) .
$$

If $\xi$ is in the first summand, we have $\operatorname{Ad}_{h^{-1}} \xi=\operatorname{Ad}_{\sigma\left(h^{-1}\right)} \xi \in \mathfrak{g}$ and therefore $\xi_{P}(p)=0$. On the other hand, if $\xi$ is in the second summand we have

$$
\operatorname{Ad}_{h^{-1}} \xi=-\operatorname{Ad}_{\sigma\left(h^{-1}\right)} \xi \in \mathfrak{p}
$$

and thus $\beta(\xi)_{h}=0$. Therefore (30) is true.

Proof of Theorem 4. The first summand in the right-hand side of (33) intersected with $\mathfrak{g}$ is $\operatorname{ker} \tau_{p}$, and the second summand intersected with $\mathfrak{g}$ is $\mathfrak{g}_{p}$. Hence ker $\tau_{p} \cap \mathfrak{g}_{p}=\{0\}$. Since $q$ is nondegenerate, $\mathfrak{g}$ and $\mathfrak{p}^{*}$ are isomorphic as vector spaces. Hence all nondegeneracy conditions are satisfied.

\section{Recovery of the original moment spaces.}

Propositions 3, 4, and 5 give equivariantly closed three-forms on each of the symmetric spaces $\mathfrak{g}^{*}, G$, and $G_{\mathbb{C}} / G$. In this section we will how the forms we have constructed here coincide with those developed independently.

Consider first $H_{0}=G \ltimes \mathfrak{g}^{*}$. The map $j_{0}: G \ltimes \mathfrak{g}^{*} \rightarrow \mathfrak{g}^{*}$, given by projection onto the $\mathfrak{g}^{*}$ factor, is right $G$-invariant. Thus it gives a left $G$-equivariant diffeomorphism between $H_{0} / G$ and $\mathfrak{g}^{*}$.

Proposition 7. We have $j_{0}^{*} \tau_{\mathfrak{g}^{*}}=\beta_{0}$, where $\beta_{0}$ is the form given by applying Proposition 5 to the Manin form $q_{0}$ on $\mathfrak{h}_{0}=\mathfrak{g} \ltimes \mathfrak{g}^{*}$.

Proof. Let $\xi \in \mathfrak{g}$ and $h=(g, \ell) \in G \ltimes \mathfrak{g}^{*}$. Then a tangent vector to $h \in H_{0}$ can be written as $L_{(g, \ell) *}(\eta, \lambda)$ for some $(\eta, \lambda) \in \mathfrak{g} \ltimes \mathfrak{g}^{*}$. We have

$$
\begin{aligned}
\left(j_{0}^{*} \tau_{\mathfrak{g}^{*}}(\xi)\right)_{(g, \ell)}\left(L_{(g, \ell) *}(\eta, \lambda)\right) & =\tau_{\mathfrak{g}^{*}}(\xi)_{\ell}\left(\left(j_{0} \circ L_{(g, \ell)}\right)_{*}(\eta, \lambda)\right) \\
& =\tau_{\mathfrak{g}^{*}}(\xi)_{\ell}\left(\operatorname{Ad}_{g}^{*} \lambda\right)=\left\langle\xi, \operatorname{Ad}_{g}^{*} \lambda\right\rangle .
\end{aligned}
$$


On the other hand,

$$
\begin{aligned}
\beta_{0}(\xi)_{(g, \ell)}\left(L_{(g, \ell) *}(\eta, \lambda)\right) & =q_{0}\left(\xi, \operatorname{Ad}_{(g, \ell)} \pi\left(L_{(g, \ell) *}(\eta, \lambda)\right)\right) \\
& =q_{0}\left(\xi, \operatorname{Ad}_{(g, \ell)}(0, \lambda)\right) \\
& =q_{0}\left((\xi, 0),\left(0, \operatorname{Ad}_{g}^{*} \lambda\right)\right)=\left\langle\xi, \operatorname{Ad}_{g}^{*} \lambda\right\rangle .
\end{aligned}
$$

Let $H_{+}=G \times G$ and define the map $j_{+}: H_{+} \rightarrow G,\left(g_{1}, g_{2}\right) \mapsto g_{1} g_{2}^{-1}$. Embed $G$ into $H_{+}$as the diagonal; it acts on $H_{+}$on the left and the right. $j_{+}$is then seen to be right $G$-invariant and thus a left $G$-equivariant diffeomorphism between $H_{+} / G$ and $G$.

Proposition 8. The map $j_{+}$pulls back the Alekseev-Meinrenken-Malkin moment form (7) to $\Xi_{+}+\beta_{+}$, the form constructed on $H_{+}$from the Manin pairing $q_{+}$.

Proof. We may write $\theta_{H_{+}}=\theta_{G}^{1}+\theta_{G}^{2}$, etc. Then for each $\left(g_{1}, g_{2}\right) \in H_{+}$,

$$
\begin{aligned}
& \left.j_{+}^{*} \theta_{G}\right|_{\left(g_{1}, g_{2}\right)}=\operatorname{Ad}_{g_{2}}\left(\theta_{G}^{1}-\theta_{G}^{2}\right) ; \\
& \left.j_{+}^{*} \bar{\theta}_{G}\right|_{\left(g_{1}, g_{2}\right)}=\operatorname{Ad}_{g_{1}}\left(\theta_{G}^{1}-\theta_{G}^{2}\right) .
\end{aligned}
$$

Therefore

$$
j_{+}^{*} \chi_{+}=j_{+}^{*} \frac{1}{12} B\left(\theta_{G},\left[\theta_{G}, \theta_{G}\right]\right)=\frac{1}{12} B\left(\theta_{G}^{1}-\theta_{G}^{2},\left[\theta_{G}^{1}-\theta_{G}^{2}, \theta_{G}^{1}-\theta_{G}^{2}\right]\right) .
$$

Now $\pi=\left(\frac{\theta_{G}^{1}-\theta_{G}^{2}}{2}, \frac{\theta_{G}^{1}-\theta_{G}^{2}}{2}\right)$, so

$$
\begin{aligned}
\Xi_{+} & =\frac{1}{3} q_{+}(\pi,[\pi, \pi]) \\
& =\frac{1}{24} q_{+}\left(\left(\theta_{G}^{1}-\theta_{G}^{2}, \theta_{G}^{1}-\theta_{G}^{2}\right),\left[\left(\theta_{G}^{1}-\theta_{G}^{2}, \theta_{G}^{1}-\theta_{G}^{2}\right),\left(\theta_{G}^{1}-\theta_{G}^{2}, \theta_{G}^{1}-\theta_{G}^{2}\right)\right]\right) \\
& =\frac{1}{24} q_{+}\left(\left(\theta_{G}^{1}-\theta_{G}^{2}, \theta_{G}^{1}-\theta_{G}^{2}\right),\left(\left[\theta_{G}^{1}-\theta_{G}^{2}, \theta_{G}^{1}-\theta_{G}^{2}\right],\left[\theta_{G}^{1}-\theta_{G}^{2}, \theta_{G}^{1}-\theta_{G}^{2}\right]\right)\right) \\
& =\frac{1}{12} B\left(\theta_{G}^{1}-\theta_{G}^{2},\left[\theta_{G}^{1}-\theta_{G}^{2}, \theta_{G}^{1}-\theta_{G}^{2}\right]\right) .
\end{aligned}
$$

Similarly,

$$
\begin{aligned}
J_{+}^{*} \tau(\xi) & =\frac{1}{2} B\left(\xi, j_{+}^{*} \theta_{G}+\bar{\theta}_{G}\right) \\
& =\frac{1}{2} B\left(\xi,\left(\operatorname{Ad}_{g_{2}}+\operatorname{Ad}_{g_{1}}\right)\left(\theta_{G}^{1}-\theta_{G}^{2}\right)\right),
\end{aligned}
$$

while

$$
\begin{aligned}
\beta_{+}(\xi) & =q_{+}\left((\xi, \xi), \operatorname{Ad}_{\left(g_{1}, g_{2}\right)}\left(\frac{\theta_{G}^{1}-\theta_{G}^{2}}{2}, \frac{\theta_{G}^{1}-\theta_{G}^{2}}{2}\right)\right) \\
& =q_{+}\left((\xi, \xi),\left(\operatorname{Ad}_{g_{1}} \frac{\theta_{G}^{1}-\theta_{G}^{2}}{2}, \operatorname{Ad}_{g_{2}} \frac{\theta_{G}^{1}-\theta_{G}^{2}}{2}\right)\right) \\
& =\frac{1}{2} B\left(\xi,\left(\operatorname{Ad}_{g_{1}}+\operatorname{Ad}_{g_{2}}\right)\left(\theta_{G}^{1}-\theta_{G}^{2}\right)\right) .
\end{aligned}
$$


Let $j_{-}: G_{\mathbb{C}} \rightarrow G_{\mathbb{C}}$ be the map $h \mapsto h \bar{h}^{-}$. Then $j_{-}$takes values in $Y$, is right $G$-invariant, and descends to a left $G$-equivariant diffeomorphism of $G_{\mathbb{C}} / G$ with $Y$.

The factor of 2 appearing in (13) is not in its original definition; it is introduced in [3] to make the theorem connecting $Y$ to $G^{*}$ more clear. Up to that same factor of 2 , we can connect our moment form on $G_{C} / G$ to that on $Y$.

Proposition 9. The map $j_{-}$pulls back the moment form $\tilde{\chi}_{Y}$ to $\Xi_{-}+\beta_{-}$, the equivariantly closed three-form on $G_{\mathbb{C}}$ arising from applying Propositions 5 and 6 to $2 q_{-}$.

Proof. Let $\theta_{G_{\mathbb{C}}}$ be the left Maurer-Cartan form, and $\widetilde{\theta}_{G_{\mathbb{C}}}$ its complex conjugate. Then for all $h \in G_{\mathbb{C}}$,

$$
\begin{aligned}
& \left.j_{-}^{*} \theta_{Y}\right|_{h}=\operatorname{Ad}_{\bar{h}}\left(\theta_{G_{\mathbb{C}}}-\tilde{\theta}_{G_{\mathbb{C}}}\right)=2 \operatorname{Ad}_{\bar{h}} \pi ; \\
& \left.j_{-}^{*} \bar{\theta}_{Y}\right|_{h}=\operatorname{Ad}_{\bar{h}}\left(\theta_{G_{\mathbb{C}}}-\tilde{\theta}_{G_{\mathbb{C}}}\right)=2 \operatorname{Ad}_{h} \pi .
\end{aligned}
$$

So

$$
\begin{aligned}
j_{-}^{*} \chi_{Y} & =\frac{1}{2} \operatorname{Im} B_{\mathbb{C}}\left(j_{-}^{*} \theta_{Y},\left[j_{-}^{*} \theta_{Y}, j_{-}^{*} \theta_{Y}\right]\right) \\
& =\frac{2}{3} \operatorname{Im} B_{\mathbb{C}}(\pi,[\pi, \pi])=\Xi_{-} .
\end{aligned}
$$

Likewise, we compute

$$
\begin{aligned}
j_{-}^{*} \tau_{Y}(\xi) & =\frac{1}{2 \sqrt{-1}} B_{\mathbb{C}}\left(\xi, j_{-}^{*} \theta_{Y}+j_{-}^{*} \bar{\theta}_{Y}\right) \\
& =\frac{1}{2 \sqrt{-1}} B_{\mathbb{C}}\left(\xi,\left(\operatorname{Ad}_{h}+\operatorname{Ad}_{\bar{h}}\right)\left(\theta_{G_{\mathbb{C}}}-\widetilde{\theta}_{G_{\mathbb{C}}}\right)\right) .
\end{aligned}
$$

Note that $\operatorname{Ad}_{h}+\operatorname{Ad}_{\bar{h}}$ is real and $\theta_{G_{\mathbb{C}}}-\widetilde{\theta}_{G_{\mathbb{C}}}$ is imaginary, so the above is in fact real. One the other hand

$$
\begin{aligned}
\beta_{-}(\xi)_{h} & =q\left(\xi, \operatorname{Ad}_{h} \pi\right)=2 \operatorname{Im} B_{\mathbb{C}}\left(\xi, \operatorname{Ad}_{h} \pi\right) \\
& =\frac{1}{\sqrt{-1}}\left(B_{C}\left(\xi, \frac{1}{2} \operatorname{Ad}_{h}\left(\theta_{G_{\mathbb{C}}}-\widetilde{\theta}_{G_{\mathbb{C}}}\right)\right)-\overline{B_{C}\left(\xi, \frac{1}{2} \operatorname{Ad}_{h}\left(\theta_{G_{\mathbb{C}}}-\widetilde{\theta}_{G_{\mathbb{C}}}\right)\right)}\right) \\
& =\frac{1}{2 \sqrt{-1}} B_{\mathbb{C}}\left(\xi,\left(\operatorname{Ad}_{\bar{h}}+\operatorname{Ad}_{h}\right)\left(\theta_{G_{\mathbb{C}}}-\widetilde{\theta}_{G_{\mathbb{C}}}\right)\right) .
\end{aligned}
$$

\section{Decompositions.}

We have shown how Manin symmetric pairs can give rise to moment spaces. We now show to extent to which the known examples of Manin symmetric pairs are the only ones.

If $G=G_{1} \times G_{2}$ is a direct product of Lie groups, and $P_{1}$ and $P_{2}$ are moment spaces for $G_{1}$ and $G_{2}$, respectively, then $P_{1} \times P_{2}$ with the equivariant form $\widetilde{\chi}_{1}+\widetilde{\chi}_{2}$ is a moment space for $G$. Thus we have a way of "building up" 
moment spaces. It is natural to try to go the other way-i.e., to decompose. We start this process at the linear level, and integrate from there.

5.1. Structure of Manin symmetric pairs. We say that a symmetric pair $(H, G)$ is Riemannian if $G$ acts by isometries on $H$. We say that a symmetric Lie algebra $(\mathfrak{h}, s)$ is orthogonal if $\mathfrak{g}=\mathfrak{h}^{s}$ is compactly embedded in $h$ and effective if $\mathfrak{g} \cap \mathfrak{h}=0$. These last two conditions are satisfied whenever $(\mathfrak{h}, s)$ is the symmetric Lie algebra associated to a Riemannian symmetric pair $(H, G)$.

Theorem 5. let $(\mathfrak{h}, s)$ be an effective, orthogonal symmetric Lie algebra with Manin pairing $q$. Then there exists a unique canonical decomposition

$$
\begin{aligned}
& \mathfrak{h}=\mathfrak{h}_{0} \oplus \mathfrak{h}_{+} \oplus \mathfrak{h}_{-} ; \\
& \mathfrak{g}=\mathfrak{g}_{0} \oplus \mathfrak{g}_{+} \oplus \mathfrak{g}_{-} ; \\
& \mathfrak{p}=\mathfrak{p}_{0} \oplus \mathfrak{p}_{+} \oplus \mathfrak{p}_{-} ;
\end{aligned}
$$$$
\text { (direct sum of ideals) }
$$$$
\text { (direct sum of ideals) }
$$

(direct sum of subspaces)

such that, with the induced symmetric and Manin pairings given by restriction, we have

$$
\begin{aligned}
\mathfrak{h}_{0} & =\mathfrak{g}_{0} \oplus \mathfrak{p}_{0} \cong \mathfrak{g}_{0} \ltimes \mathfrak{g}_{0}^{*} ; \\
\mathfrak{h}_{+} & =\mathfrak{g}_{+} \oplus \mathfrak{p}_{+} \cong \mathfrak{g}_{+} \times \mathfrak{g}_{+} ; \\
\mathfrak{h}_{-} & =\mathfrak{g}_{-} \oplus \mathfrak{p}_{-} \cong \mathfrak{g}_{-} \otimes \mathbb{C}
\end{aligned}
$$

These isomorphisms are in fact isometries with respect to $q$.

We will prove this in a series of lemmas. To begin, assume that $\mathfrak{g}$ is simple. Let $\kappa$ be the negative of the Killing form on $\mathfrak{h}$. Then $\kappa$ is positive-definite on $\mathfrak{g}, \operatorname{ad}_{\mathfrak{h}}$-invariant, and

$$
\kappa\left(s \zeta_{1}, \zeta_{2}\right)=\kappa\left(\zeta_{1}, \zeta_{2}\right)
$$

or,

$$
\kappa\left(s \zeta_{1}, \zeta_{2}\right)=\kappa\left(\zeta_{1}, s \zeta_{2}\right),
$$

for all $\zeta_{1}, \zeta_{2} \in \mathfrak{h}$. Define $J: \mathfrak{h} \rightarrow \mathfrak{h}$ by

$$
q\left(J \zeta_{1}, \zeta_{2}\right)=\kappa\left(\zeta_{1}, \zeta_{2}\right) .
$$

Lemma 2. (a) The map $J$ commutes with the adjoint action of $\mathfrak{h}$ on itself. That is, for all $\zeta \in \mathfrak{h}$,

$$
J \circ \operatorname{ad}_{\zeta}=\operatorname{ad}_{\zeta} \circ
$$

or, for all $\zeta_{1}$ and $\zeta_{2}$,

$$
J\left[\zeta_{1}, \zeta_{2}\right]=\left[J \zeta_{1}, \zeta_{2}\right]
$$

(b) The map $J$ anticommutes with $s: J \circ s=-s \circ J$. So $J$ takes $\mathfrak{g}$ into $\mathfrak{p}$ and vice versa.

(c) The map $J$ is self-adjoint with respect to $q$. 
(d) The restriction $\left.J\right|_{\mathfrak{g}}$ is a vector space isomorphism $\mathfrak{g} \cong \mathfrak{p}$.

Proof. The first two parts are straightforward. The third is a simple consequence of the symmetry of $\kappa$ and of $q$. The last follows from the fact that $\kappa$ is positive definite on $\mathfrak{g}$.

It follows that $J^{2}$ is an endomorphism of $\mathfrak{g}$ as a $\mathfrak{g}$-module. By Lemma 2, Part (c), $J^{2}$ is self-adjoint. Therefore, $\mathfrak{g}$ has an orthonormal basis of eigenvectors with real eigenvalues. Since each eigenspace is an ideal of $J^{2}$, it follows by simplicity that $\mathfrak{g}=\mathfrak{g}_{\lambda}$ is a single eigenspace. Thus for all $\xi, \eta \in \mathfrak{g}$,

$$
[J \xi, J \eta]=J^{2}[\xi, \eta]=\lambda[\xi, \eta] .
$$

If $\lambda=0$, then $\mathfrak{p}$ is an abelian ideal of $\mathfrak{h}$ dual by $q$ to $\mathfrak{g}$, and hence $\mathfrak{h} \cong \mathfrak{g} \ltimes \mathfrak{g}^{*}$. Otherwise, the endomorphism $\frac{1}{\sqrt{|\lambda|}} J$ enjoys all the properties of Lemma 2, so we may assume that $|\lambda|=1$. If $\lambda=1$, the map

$$
\begin{aligned}
T_{+}: \mathfrak{h} & \longrightarrow \mathfrak{g} \times \mathfrak{g} ; \\
(\xi, J \eta) & \longmapsto \frac{1}{2}(\xi+\eta, \xi-\eta)
\end{aligned}
$$

is an isomorphism of $(\mathfrak{h}, s, q)$ onto $\left(\mathfrak{h}_{+}, s_{+}, q_{+}\right)$. On the other hand if $\lambda=-1$ the map

$$
\begin{aligned}
T_{-}: \mathfrak{h} & \longrightarrow \mathfrak{g} \otimes \mathbb{C} ; \\
(\xi, J \eta) & \longmapsto \xi+\sqrt{-1} \eta
\end{aligned}
$$

is an isomorphism onto $\left(\mathfrak{h}_{-}, s_{-}, q_{-}\right)$. This concludes the proof of Theorem 5 in the case that $\mathfrak{g}$ is simple.

Now if $\mathfrak{h}$ is effective, then $\mathfrak{g}$ is at least semisimple. Therefore we have a decomposition

$$
\mathfrak{g}=\bigoplus_{\lambda \in \Sigma} \mathfrak{g}_{\lambda}
$$

where $\Sigma$ is the set of eigenvalues of $J^{2}$. The eigenspaces $\mathfrak{g}_{\lambda}$ are ideals of $\mathfrak{g}$. For each $\lambda$, let $\mathfrak{p}_{\lambda}=J \mathfrak{g}_{\lambda}$. 
Lemma 3. For each pair of eigenvalues $(\lambda, \mu)$, the following commutation relations hold:

(a)

$$
\left[\mathfrak{g}_{\lambda}, \mathfrak{g}_{\mu}\right] \subseteq \begin{cases}0 & \text { if } \lambda \neq \mu \\ \mathfrak{g}_{\lambda} & \text { if } \lambda=\mu\end{cases}
$$

(b)

$$
\left[\mathfrak{g}_{\lambda}, \mathfrak{p}_{\mu}\right] \subseteq \begin{cases}0 & \text { if } \lambda \neq \mu \\ \mathfrak{p}_{\lambda} & \text { if } \lambda=\mu\end{cases}
$$

(c)

$$
\left[\mathfrak{p}_{\lambda}, \mathfrak{p}_{\mu}\right] \subseteq \begin{cases}0 & \text { if } \lambda \neq \mu \\ \mathfrak{g}_{\lambda} & \text { if } \lambda=\mu\end{cases}
$$

Proof.

(a) Let $\xi \in \mathfrak{g}_{\lambda}$ and $\eta \in \mathfrak{g}_{\mu}$. Then since $J^{2}$ is a $\mathfrak{g}$-module homomorphism, we have that $[\xi, \eta] \in \mathfrak{g}_{\lambda} \cap \mathfrak{g}_{\mu}$.

(b) Given $\xi$ and $\eta$ as above, notice

$$
[\xi, J \eta]=J[\xi, \eta] \in J\left[\mathfrak{g}_{\lambda}, \mathfrak{g}_{\mu}\right] .
$$

(c) Finally,

$$
[J \xi, J \eta]=J^{2}[\xi, \eta] \in\left[\mathfrak{g}_{\lambda}, \mathfrak{g}_{\mu}\right]
$$

This shows that each $\mathfrak{h}_{\lambda}=\mathfrak{g}_{\lambda} \oplus \mathfrak{p}_{\lambda}$ is an ideal of $\mathfrak{h}$. Each $\mathfrak{h}_{\lambda}$ is isomorphic to one of the three canonical types, and we can collect them by type. This proves Theorem 5 .

Theorem 6. Let $(H, G)$ be a Manin symmetric pair, with $G$ semisimple and $H$ connected and simply connected. Then the moment space $P=H / G$ has a decomposition

$$
P=P_{0} \times P_{+} \times P_{-}
$$

and $G$ has a decomposition

$$
G=G_{0} \times G_{+} \times G_{-}
$$

such that $P_{0}$ is a moment space for $G_{0}$ isomorphic to $\mathfrak{g}_{0}^{*}$, $P_{+}$is a moment space for $G_{+}$isomorphic to $G_{+}$, and $P_{-}$is a moment space for $G_{-}$isomorphic to $\left(G_{-}\right)_{\mathbb{C}} / G_{-}$. 
Proof. It follows from the homotopy exact sequence for the fibration $G \rightarrow$ $H \rightarrow H / G$ that if $H$ is simply connected and $G$ is connected, then $H / G$ is simply connected.

Since $G$ is semisimple, $\mathfrak{h}$ is an effective, orthogonal, Manin symmetric Lie algebra. Therefore, we can decompose $\mathfrak{h}$ as in Theorem 5 into $\mathfrak{h}=$ $\mathfrak{h}_{0} \oplus \mathfrak{h}_{+} \oplus \mathfrak{h}_{-}$. Let $H_{0} \times H_{+} \times H_{-}$be the corresponding decomposition of $H$. Likewise $\mathfrak{g}$ decomposes and we can write $G=G_{0} \times G_{+} \times G_{-}$. Then

$$
P=H / G=\frac{H_{0} \times H_{+} \times H_{-}}{G_{0} \times G_{+} \times G_{-}}=H_{0} / G_{0} \times H_{+} / G_{+} \times H_{-} / G_{-} .
$$

5.2. Relaxing $\boldsymbol{H}$-invariance. This shows that we have exhausted all possibilities of creating moment spaces from Manin symmetric pairs, once we allow suitable assumptions about semisimplicity and connectedness. In fact, we can relax one of the conditions of a Manin form, weakening a hypothesis in Theorem 5, and thus arriving at a stronger Theorem 6 .

Let $(H, G)$ be any symmetric pair such that $H / G$ is a moment space. Let $\tilde{\chi}=\chi+\tau$ be the equivariantly closed three-form. Then $\tau$ pulls back to a linear map $\beta: \mathfrak{g} \rightarrow \Omega^{1}(H)^{G^{\text {op }}}$, where we use the op-superscript to denote the right action of $G$ on $H$. Evaluating $\beta$ at the identity of $H$ gives a map $b: \mathfrak{g} \rightarrow \mathfrak{h}^{*}$. Notice that for $g \in G, \xi \in \mathfrak{g}$, and $\zeta \in \mathfrak{h}$,

$$
b\left(\operatorname{Ad}_{g}, \xi, \zeta\right)=\beta\left(\operatorname{Ad}_{g} \xi\right)_{e}(\zeta) .
$$

By left-equivariance of $\beta$, we have

$$
=\beta(\xi)_{g^{-1}}\left(L_{g^{-1}} \zeta\right) .
$$

Because $\beta$ is right-invariant, this is

$$
\begin{aligned}
& =\beta(\xi)_{e}\left(R_{g_{*}} L_{g^{-1} *} \zeta\right) \\
& =b\left(\xi, \operatorname{Ad}_{g^{-1}} \zeta\right) .
\end{aligned}
$$

Furthermore, again by right-invariance,

$$
\begin{aligned}
b(\xi, \eta) & =\beta(\xi)_{e}(\eta) \\
& =\left\langle\beta(\xi), \eta_{R}\right\rangle_{e} \\
& =0 .
\end{aligned}
$$

Since $\mathfrak{h}$ is symmetric and has a canonical decomposition, we can uniquely extend $b$ to an inner product $q$ on $\mathfrak{h}$ with respect to which $\mathfrak{g}$ and $\mathfrak{p}$ are dual isotropic subspaces and $s$ is skew-symmetric. This form is not necessarily completely $\mathfrak{h}$-invariant, however, only $\mathfrak{g}$-invariant. Nevertheless, this suffices.

Theorem 7. Let $(\mathfrak{h}, s, q)$ be an effective orthogonal symmetric Lie algebra, with $\mathfrak{g}$ semisimple and $q$ a Manin pairing assumed to be only $\mathfrak{g}$-invariant. Then there is a canonical decomposition of $\mathfrak{h}$ as in Theorem 5. 
Then we can immediately, using techniques similar to Theorem 6 , prove:

Theorem 8. let $(H, G)$ be a Riemannian symmetric pair, with $G$ connected and $H$ simply connected. Suppose that $H / G$ is a moment space for $G$. Then there is a decomposition of $H, H$, and $H / G$ as in Theorem 6.

Proof of Theorem 7. Assume that $\mathfrak{g}$ is simple, and complexify $\mathfrak{h}, \mathfrak{g}, s$, and $q$. Then $J: \mathfrak{g} \rightarrow \mathfrak{p}$ can still by constructed by $q(J \xi, \eta)=\kappa(\xi, \eta)$. Define for $\xi, \eta \in \mathfrak{g}$,

$$
\{\xi, \eta\} \stackrel{\text { def }}{=}[J \xi, J \eta]
$$

Then clearly

$$
\left[\xi_{1},\left\{\xi_{2}, \xi_{3}\right\}\right]=\left\{\left[\xi_{1}, \xi_{2}\right], \xi_{3}\right\}+\left\{\xi_{2},\left[\xi_{1}, \xi_{3}\right]\right\} .
$$

Hence $\{\cdot, \cdot\}$ is a homomorphism of $\mathfrak{g}$-modules. For simple Lie algebras, however, all such homomorphisms are scalar multiples of the Lie bracket (see below). Thus there exists a complex number $\lambda$ such that $\{\xi, \eta\}=\lambda[\xi, \eta]$ for all $\xi$ and $\eta$. But since $J$ is real, $\lambda$ must be real, too, and we are in the same situation as in Theorem 5 .

It remains to prove that

$$
\operatorname{Hom}_{\mathfrak{g}}\left(\bigwedge^{2} \mathfrak{g}, \mathfrak{g}\right)=\mathbb{C}[\cdot, \cdot]
$$

Since $\mathfrak{g}$ is simple, it is enough to show that $\left(\Lambda^{2} \mathfrak{g}\right)_{\text {ad }}=\mathfrak{g}$, where for any $\mathfrak{g}$ module $M, M_{\text {ad }}$ denotes the ad-primary component of $M$. Though $\kappa$ we may identify $\mathfrak{g}^{*} \cong \mathfrak{g}$; thus the algebra $\Lambda \mathfrak{g}$ (on which $\mathfrak{g}$ acts preserving the grading) has a differential $d$ which is also a $\mathfrak{g}$-module homomorphism. Then as shown by Kostant [7, Theorems D and E], $(\bigwedge \mathfrak{g})_{\text {ad }}=A_{\text {ad }} \otimes(\bigwedge \mathfrak{g})^{\mathfrak{g}}$, where $A$ is the exterior subalgebra generated by the image of $d^{1} \mathfrak{g} \rightarrow \bigwedge^{2} \mathfrak{g}$. By restricting to the degree two subspace, we see that $\left(\Lambda^{2} \mathfrak{g}\right)_{\text {ad }}$ is the image of $d^{1}$. But since $H^{1}(\mathfrak{g}, \mathbb{C})=0, d^{1}$ is injective, and so the image of $d^{1}$ is isomorphic to $\mathfrak{g}$.

\section{References}

[1] A.Yu. Alekseev, On Poisson actions of compact Lie groups on symplectic manifolds, J. Differential Geom., 45(2) (1997), 241-256, MR 99b:58086, Zbl 0912.53018.

[2] A. Alekseev and Y. Kosmann-Schwarzbach, Manin pairs and moment maps, J. Differential Geom., 56(1) (2000), 133-165, CMP 1863024.

[3] A. Alekseev, A. Malkin and E. Meinrenken, Lie group valued moment maps, J. Differential Geom., 48(3) (1998), 445-495, MR 99k:58062, Zbl 0948.53045.

[4] M.F. Atiyah and R. Bott, The moment map and equivariant cohomology, Topology, 23(1) (1984), 1-28, MR 85e:58041, Zbl 0521.58025. 
[5] V. Guillemin and S. Sternberg, Symplectic Techniques in Physics, second ed., Cambridge University Press, Cambridge, 1990, MR 91d:58073, Zbl 0734.58005.

[6] S. Helgason, Differential Geometry, Lie Groups and Symmetric Spaces, Academic Press Inc. [Harcourt Brace Jovanovich Publishers], New York, 1978, MR 80k:53081, Zbl 0451.53038.

[7] B. Kostant, Clifford algebra analogue of the Hopf-Koszul-Samelson theorem, the $\rho$ decomposition $C(\mathfrak{g})=$ End $V_{\rho} \otimes C(P)$, and the $\mathfrak{g}$-module structure of $\bigwedge \mathfrak{g}$, Adv. Math., 125(2) (1997), 275-350, MR 98k:17009, Zbl 0882.17002.

[8] M. Leingang, Symmetric pairs and moment spaces, math.SG/9810064.

[9] _ Symmetric Space Valued Moment Maps, Ph.D. Thesis, Harvard, 2000.

[10] J.-H. Lu, Momentum mappings and reduction of Poisson actions, in 'Symplectic geometry, groupoids, and integrable systems' (Berkeley, CA, 1989), 209-226, Math. Sci. Res. Inst. Publ., 20, Springer, New York, 1991, MR 92j:58036, Zbl 0735.58004.

[11] J.-H. Lu and A. Weinstein, Poisson Lie groups, dressing transformations, and Bruhat decompositions, J. Differential Geom., 31(2) (1990), 501-526, MR 91c:22012, Zbl 0673.58018.

Received April 27, 2001 and revised October 21, 2002.

Department of Mathematics

HARVARD UNIVERSITY

SCIENCE CEnTER 325

1 OxFord StreEt

CAmbridge, MA 02138

E-mail address: leingang@math.harvard.edu 